\title{
Markov-Kakutani Theorem on Hyperspace of a Banach Space
}

\author{
Jennifer Shueh-Inn Hu and Thakyin $\mathrm{Hu}$
}

\begin{abstract}
Suppose $X$ is a Banach space and $K$ is a compact convex subset of $X$. Let $\mathcal{F}$ be a commutative family of continuous affine mappings of $K$ into $K$. It follows from MarkovKakutani Theorem that $\mathcal{F}$ has a common fixed point in $K$. Suppose now $(C C(X), h)$ is the corresponding hyperspace of $X$ containing all compact, convex subsets of $X$ endowed with Hausdorff metric $h$. We shall prove the above version of Markov-Kakutani Theorem is valid on the hyperspace $(C C(X), h)$.
\end{abstract}

\section{Introduction}

Suppose $X$ is a Banach space and $C C(X)$ is the collection of all non-empty compact, convex subsets of $X$ endowed with the Hausdorff metric $h$. $(C C(X), h)$ is known as the hyperspace over $X$. Blaschke [1] proved that a bounded sequence $\left\{A_{n}\right\}$ of $\left(C C\left(\mathbb{R}^{k}\right), h\right)$ has a subsequence $\left\{A_{n_{i}}\right\}$ such that $A_{n_{i}} \stackrel{h}{\rightarrow} A_{0}$ (or $h\left(A_{n_{i}}, A_{0}\right) \rightarrow 0$ as $\left.i \rightarrow \infty\right)$ for some $A_{0} \in C C\left(\mathbb{R}^{k}\right)$. This result is an extension of the classical Bolzano-Weierstrass Theorem.

De Blasi and Myjak [3] introduced the concept of sequential convergence by means of usual support functionals $\sigma_{A}\left(x^{*}\right)=\sup \left\{x^{*}(x): x \in A\right\}$ and proved an infinite dimensional version of Blaschke's Theorem. Motivated by the above mentioned papers, we try to improve the above results. However, we find that the traditional support functional techniques do not work, hence we have to develop some new techniques which are very useful to attack problems on $(C C(X), h)$. We are able to prove a theorem that generalizes all the above mentioned results [2]. We may also use the above techniques to generalize Brodskii-Milman's [6] and Browder-Kirk's [4] fixed point theorems. We then turn our investigation to Markov-Kakutani's fixed point theorem.

Recall that Markov-Kakutani's fixed point theorem states that if $K$ is a compact, convex subset of $X$ and $\mathcal{F}$ is a commutative family of continuous affine mappings of $K$ into $K$, then $\mathcal{F}$ has a common fixed point in $K$. However, since there is no subtraction on the hyperspace $(C C(X), h)$

2010 Mathematics Subject Classification. 47H10, 54A05, 54B20.

Key words and phrases. Markov-Kakutani theorem, Common fixed point, Hyperspace. Corresponding author: Jennifer Shueh-Inn Hu. 
to simplify calculations, we face difficulty when we try to prove the theorem. This difficulty is overcome when we come up with a simple lemma that solves our problems and enables us to prove that Markov-Kakutani Theorem can be extended to the hyperspace $(C C(X), h)$, which is the main result of this paper. Examples have been given showing that some results that are valid on the underlying Banach space $X$ cannot be extended to the hyperspace $(C C(X), h)[3,5]$. Our theorem might provide a new tool to help solve the above mentioned problems.

\section{Notations and Preliminaries}

In the following we shall define the basic concepts, state and prove some basic properties for completeness.

(i) For $A, B \in C C(X)$, let $N(A, \epsilon)=\{x \in X: d(x, a)<\epsilon$ for some $a \in A\}$, define $h(A, B)=\inf \{\epsilon>0: A \subset N(B, \epsilon), B \subset N(A, \epsilon)\}$. The function $h$ is known as the Hausdorff metric and $(C C(X), h)$ is known as the hyperspace over $X$.

(ii) $A+B=\{a+b: a \in A, b \in B\}, \lambda A=\{\lambda a: a \in A\}$.

(iii) $\Omega \subseteq C C(X)$ is said to be convex if $A_{i} \in C C(X), \alpha_{i} \geq 0, \sum_{i=1}^{n} \alpha_{i}=1$ implies $\sum_{i=1}^{n} \alpha_{i} A_{i} \in \Omega$,

(iv) $T: C C(X) \rightarrow C C(X)$ is affine if $T(\alpha A+\beta B)=\alpha T(A)+\beta T(B)$ where $\alpha, \beta \geq$ $0, \alpha+\beta=1$.

(v) $[A, B]=\{\alpha A+(1-\alpha) B: 0 \leq \alpha \leq 1\}$ is the line segment joining $A$ and $B$.

Next we shall state the following basic properties of the Hausdorff metric $h$ in the following lemma and prove some of them.

\section{Lemma 2.1.}

(a) $A, B \in C C(X), \lambda>0$ implies $A+B, \lambda A \in C C(X)$.

Proof. Since addition and scalar multiplication are continuous.

(b) $A \in C C(X), \lambda, \mu>0$ implies $(\lambda+\mu) A=\lambda A+\mu A$.

Proof. Omitted.

(c) $h(\lambda A, \lambda B)=\lambda h(A, B), \lambda>0$. 
Proof. Omitted.

(d) $h(A+C, B+D) \leq h(A, B)+h(C, D)$.

Proof. Let $\epsilon_{1}>h(A, B), \epsilon_{2}>h(C, D)$ and $\epsilon=\epsilon_{1}+\epsilon_{2}$. Recall that $h(A+C, B+D)=\inf \{\epsilon>0: A+C \subseteq N(B+D, \epsilon), B+D \subseteq N(A+C, \epsilon)\}$. If $a+c \in A+C$ is given, then it follows from $h(A, B)<\epsilon_{1}, h(C, D)<\epsilon_{2}$ that there exists $b \in B$ such that $\|a-b\|<\epsilon_{1}$ and $\|c-d\|<\epsilon_{2}$. Hence $\|(a+c)-(b+d)\| \leq$ $\|(a-b)+(c-d)\| \leq\|a-b\|+\|c-d\|<\epsilon_{1}+\epsilon_{2}=\epsilon$. Thus $A+C \subseteq N(B+D, \epsilon)$. Similarly, $B+D \subseteq N(A+C, \epsilon)$. Consequently $h(A+C, B+D) \leq h(A, B)+h(C, D)$.

(e) $h(A+C, B+C) \leq h(A, B)$.

Proof. Let $h(A, B)<\epsilon$. Then we have $A \subset N(B, \epsilon)$, and $B \subset N(A, \epsilon)$. To show $A+C \subseteq$ $N(B+C, \epsilon)$ and $B+C \subseteq N(A+C, \epsilon)$, we let $a+c \in A+C$. Since $A \subset N(B, \epsilon)$, there exists $b \in B$ such that $\|a-b\|<\epsilon$, choose $c \in C$ as the given $c$. And we have $b+c \in B+C$ with $\|(a+c)-(b+c)\|=\|a-b\|<\epsilon$ which in turn implies that $(a+c) \in N(B+C, \epsilon)$ or $A+C \subseteq N(B+C, \epsilon)$. Similarly, we can prove that $B+C \subseteq N(A+C, \epsilon)$. Consequently, $h(A+C, B+C)=\inf \{\epsilon>0: A+C \subset N(B+C, \epsilon)$ and $B+C \subseteq N(A+C, \epsilon)\} \leq$ $h(A, B)$ and the proof is complete.

(f) Addition and scalar multiplication by non-negative scalars are continuous operations on the hyperspace $(C C(X), h)$.

Proof. Let $\mathcal{U}_{h}(A, \epsilon)=\{B \in C C(X): h(A, B)<\epsilon\}$. Suppose $A, B \in C C(X)$ and $\mathcal{U}_{h}(A+B, \epsilon)$ is an open set containing $A+B$. Let $\mathcal{U}_{h}(A, \epsilon / 4), \mathcal{U}_{h}(B, \epsilon / 4)$ be open neighborhoods of $A$ and $B$. Let $C \in \mathcal{U}_{h}(A, \epsilon / 4)$ and $D \in \mathcal{U}_{h}(B, \epsilon / 4)$. Then it follows from (d) that

$$
h(A+B, C+D) \leq h(A, C)+h(B, D)<\epsilon / 4+\epsilon / 4=\epsilon / 2<\epsilon,
$$

showing that addition is continuous. To show scalar multiplication is continuous, let $\mathcal{U}_{h}(\alpha A, \epsilon)$ be given with $\alpha>0$ and $0<\beta<\alpha$ (the case $\beta>\alpha$ will be treated similarly and thus omitted). Let $\gamma \in(\beta, \alpha)$. Then $(\alpha-\gamma)$ and $\gamma$ are both positive and we may use (b), (c) and (d) to get

$$
\begin{aligned}
h(\gamma B, \alpha A) & =h(\gamma B,[(\alpha-\gamma)+\gamma] A) \\
& \leq h(\gamma B, \gamma A)+h(\{0\},(\alpha-\gamma) A) \\
& =\gamma h(B, A)+h(\{0\},(\alpha-\gamma) A) \\
& =\gamma h(B, A)+(\alpha-\gamma) h(\{0\}, A) .
\end{aligned}
$$


Choose $\mathcal{U}_{h}(A, \epsilon /(4 \gamma))$ and $\alpha-\gamma<\epsilon /(4 h(\{0\}, A))$. Consequently for $B \in \mathcal{U}_{h}(A, \epsilon /(4 \gamma))$ and $\beta<\gamma<\alpha$, we have

$$
h(\gamma B, \alpha A) \leq \epsilon / 4+\epsilon / 4=\epsilon / 2<\epsilon,
$$

which implies that scalar multiplication is continuous.

Now that we have all the essential tools, we are ready to state and prove our main result on the hyperspace $(C C(X), h)$.

\section{Main Result}

Theorem 3.1. Suppose $X$ is a Banach space, $(C C(X), h)$ is the corresponding hyperspace endowed with Hausdorff metric $h$. Let $\mathcal{F}:(C C(X), h) \rightarrow(C C(X), h)$ be a commutative family of continuous affine mappings and $\Omega \subseteq(C C(X), h)$ be an h-compact, convex subset of $(C C(X), h)$. Suppose $\Omega$ is invariant under $\mathcal{F}$, i.e. $\mathcal{F}(\Omega) \subseteq \Omega$ or $f(\Omega) \subset \Omega$ for each $f \in \mathcal{F}$. Then $\mathcal{F}$ has a common fixed point in $\Omega$.

Proof. For each $T \in \mathcal{F}$ and each positive integer $n$, define

$$
T_{n} A=\frac{1}{n} \sum_{k=0}^{n-1} T^{k} A
$$

where $T^{0}=$ identity. It follows from Lemma 2.1 (f) that addition and scalar multiplication are continuous operations. Also $f$ is affine and continuous. Thus each $T_{n}$ is $h$-continuous and $T_{n}(\Omega) \subseteq \Omega$. The commutativity of $\mathcal{F}$ implies that $T_{n}\left(S_{m}(\Omega)\right)=S_{m}\left(T_{n}(\Omega)\right) \subseteq T_{n}(\Omega) \cap S_{m}(\Omega)$. Consequently, $\left\{T_{n}(\Omega): n=1,2, \cdots ; T \in \mathcal{F}\right\}$ is a family of $h$-compact convex subsets of $\Omega$ with finite intersection property. Thus $\cap T_{n}(\Omega) \neq \emptyset$ where the intersection is taken over $n=1,2, \cdots$, and all $T \in \mathcal{F}$. Let $A_{0}=\cap T_{n}(\Omega)$. We claim that $A_{0}$ is a common fixed point. Otherwise, $h\left(T A_{0}, A_{0}\right) \neq 0$ and we may let $h\left(T A_{0}, A_{0}\right)=\delta>0$. Note that for each $n, A_{0} \in T_{n}(\Omega)$ implies that there is some $B_{n} \in \Omega$ with

$$
A_{0}=\frac{1}{n} \sum_{k=0}^{n-1} T^{k}\left(B_{n}\right)=\frac{1}{n}\left(B_{n}+T B_{n}+\cdots+T^{n-1} B_{n}\right),
$$

and

$$
T A_{0}=\frac{1}{n} \sum_{k=1}^{n} T^{k}\left(B_{n}\right)=\frac{1}{n}\left(T B_{n}+\cdots+T^{n-1} B_{n}+T^{n} B_{n}\right) .
$$

Note that the two expressions of $A_{0}$ and $T A_{0}$ have many terms in common and we may usually use subtraction to simplify them in case they are points of $X$. However, they are compact, convex 
subsets and we do not have subtraction on the hyperspace $(C C(X), h)$. Fortunately, we have (e) to help us. Thus, since $\Omega$ is compact,

$$
\begin{aligned}
h\left(A_{0}, T A_{0}\right) & =h\left(\frac{B_{n}+T B_{n}+\cdots+T^{n-1} B_{n}}{n}, \frac{T B_{n}+\cdots+T^{n-1} B_{n}+T^{n} B_{n}}{n}\right) \\
& \leq \frac{h\left(B_{n}, T^{n} B_{n}\right)}{n} \\
& \leq \frac{1}{n} \operatorname{diam}(\Omega) \\
& <\infty .
\end{aligned}
$$

Since $n$ can be any positive integer, we may choose $n$ large enough so that $\operatorname{diam}(\Omega) / n<\delta / 2$. That is a contradiction. Consequently, $A_{0}=T A_{0}$ and the proof is complete.

\section{References}

[1] W. Blaschke, Kreis und Kugel, Verlag von Veit \& Co., Leipzig, 1916.

[2] F. S. De Blasi, T. Hu and J.-C. Huang, Weak*-topology and Alaoglu's theorem on hyperspace, Journal of Nonlinear and Convex Analysis, 10 (2009), 33-40.

[3] F. S. De Blasi and J. Myjak, Weak convergence of convex sets in Banach spaces, Archiv der Mathematik, 47 (1986), 448-456.

[4] T. Hu and J. C. Fang, Weak topology and Browder-Kirk's theorem on hyperspace, Journal of Mathematical Analysis and Applications, 334 (2007), 799-803.

[5] Jennifer S.-I. Hu and T. Hu, Krein-Milman's extreme point theorem and weak topology on hyperspaces, Taiwanese Journal of Mathematics, 20 (2016), 629-638.

[6] T. Hu and J. C. Huang, Weak and weak ${ }^{*}$ topologies and Brodskii-Milman's theorem on hyperspaces, Taiwanese Journal of Mathematics, 13 (2009), 459-466.

Shueh-Inn Hu Department of Applied Statistics and Information Science, Ming Chuan University, Guishan, Taoyuan, Taiwan

E-mail: jenhu@mail.mcu.edu.tw

Thakyin Hu Department of Mathematics, Tamkang University, Tamsui, Taipei, Taiwan E-mail: 026968@mail.tku.edu.tw 